\title{
Establishment of a PEG-mediated protoplast transformation system based on DNA and CRISPR/Cas9 ribonucleoprotein complexes for banana
}

Shaoping $\mathrm{Wu}^{1,2+}$, Haocheng $\mathrm{Zhu}^{3,4+}$, Jinxing $\mathrm{Liu}^{3}$, Qiaosong Yang ${ }^{2}$, Xiuhong Shao ${ }^{2}$, Fangcheng $\mathrm{Bi}^{2}$, Chunhua $\mathrm{Hu}^{2}$, Heqiang Huo ${ }^{5}$, Kunling Chen ${ }^{3^{*}}$ and Ganjun $\mathrm{Yi}^{2^{*}}$

\begin{abstract}
Background: To date, CRISPR/Cas9 RNP editing tools have not been applied to the genetic modification of banana. Here, the establishment of a PEG-mediated banana protoplast transformation system makes it possible to build an efficient DNA-free method for a site-directed mutagenesis system.

Results: Protoplasts constitute a versatile platform for transient expression in plant science. In this study, we established a PEG-mediated banana protoplast transformation system. This system was further optimized for successfully delivering CRISPR/Cas9 and CRISPR/Cas12a plasmids and CRISPR/Cas9 ribonucleoproteins (RNPs) for targeted delivery of the PDS gene into banana protoplasts. Specific bands were observed in PCR-Restriction Enzyme Digestion (PCR-RE) assays, and Sanger sequencing of single clones further confirmed the occurrence of indels at target sites. Deep amplicon sequencing results showed that the editing efficiency of the CRISPR/Cas9 system was higher than that of the other two systems.

Conclusions: The PEG-mediated banana protoplast transformation system can serve as a rapid and effective tool for transient expression assays and sgRNA validation in banana. The application of the CRISPR/Cas9 RNP system enables the generation of banana plants engineered by DNA-free gene editing.
\end{abstract}

Keywords: PEG-mediated, Protoplast transformation, Deep amplicon sequencing, Genome editing, DNA-free

\footnotetext{
*Correspondence: klchen@genetics.ac.cn; yiganjun@vip.163.com

†Shaoping Wu and Haocheng Zhu contributed equally to this work.

${ }^{3}$ State Key Laboratory of Plant Cell and Chromosome Engineering, Center for Genome Editing, Institute of Genetics and Developmental Biology, Chinese Academy of Sciences, Beijing, China

${ }^{2}$ Key Laboratory of South Subtropical Fruit Biology and Genetic Resource Utilization (Ministry of Agriculture and Rural Affairs), Guangdong Key Laboratory of Tropical and Subtropical Fruit Tree Research, Institute of Fruit Tree Research, Guangdong Academy of Agricultural Sciences, Guangzhou, China

Full list of author information is available at the end of the article
}

(c) The Author(s). 2020 Open Access This article is licensed under a Creative Commons Attribution 4.0 International License, which permits use, sharing, adaptation, distribution and reproduction in any medium or format, as long as you give appropriate credit to the original author(s) and the source, provide a link to the Creative Commons licence, and indicate if changes were made. The images or other third party material in this article are included in the article's Creative Commons licence, unless indicated otherwise in a credit line to the material. If material is not included in the article's Creative Commons licence and your intended use is not permitted by statutory regulation or exceeds the permitted use, you will need to obtain permission directly from the copyright holder. To view a copy of this licence, visit http://creativecommons.org/licenses/by/4.0/. The Creative Commons Public Domain Dedication waiver (http://creativecommons.org/publicdomain/zero/1.0/) applies to the data made available in this article, unless otherwise stated in a credit line to the data. 


\section{Background}

A protoplast is a cell of a plant, fungus, bacterium, or archaeon from which the cell wall has been removed by plasmolysis, leaving the protoplasm and plasma membrane. As early as 1892, Klercker obtained protoplasts by a mechanical method [1], which resulted in a low yield and suffered from difficult operation and poor applicability. In 1960, Cocking successfully isolated tomato root tip protoplasts for the first time by enzymatic hydrolysis [2]. This method was widely used because of its high yield, high activity, easy operation and wide adaptability. The transient transformation system of plant protoplasts without unique cell wall characteristics is extensively used in genetic research involving gene function identification, subcellular localization and gene editing. The common methods used for plant protoplast transformation include PEG-mediated transformation [3, 4], electroporation-mediated transformation [5-9] and microinjection-based transformation [10]. Among these, the PEG-mediated method is widely used due to its easy operation, low cost, lack of requirements for specific equipment and generation of stable results. To this date, mature and stable genetic transformation systems for protoplast transient expression have been established in Arabidopsis [11-14], wheat [15], rice [16], maize [17] and other species.

Currently, there are many reports on PEG-mediated protoplast transformation of DNA, but few reports on PEG-mediated protoplast transformation of ribonucleoprotein (RNP). In 2015, Woo et al. directly transferred RNP into the protoplasts of Arabidopsis thaliana, tobacco and rice for the first time. The genome-edited mutant regenerated from protoplasts contained no transgenic ingredients [18]. In 2016, Malnoy et al. successfully transferred RNP into protoplasts from grapes and apples, and the mutation efficiency was as high as $6.9 \%$ [19]. To date, several investigations have reported the application of CRISPR-Cas9 gene editing technology in bananas [20-23], yet transgenic plants containing TDNA were generated in each of these cases of CRISPR/ Cas9 gene editing, and a DNA-free genome editing method has not been developed in banana. In this study, a PEG-mediated transformation system was established, which provided an effective method for the detection of gRNA activity. This system was used to successfully deliver RNPs into banana protoplasts, and the RNP system was detected to be working through deep amplicon sequencing, laying a good foundation for the further study of banana genome editing.

\section{Results}

Establishment of a PEG-mediated protoplast transformation system in banana

To establish a PEG-mediated banana protoplast transformation system, we optimized the transformation method based on transformation protocols for rice and wheat. The rice and wheat protocols are quite effective in rice and wheat protoplast transformation, and transformation efficiency reaches 58.4 and $64.5 \%$, respectively, confirmed by flow cytometry detection [24]. The transformation efficiency of this protocol in banana was much lower than for rice or wheat; after 5-day incubation in darkness only a few GFP fluorescent spots appeared (Fig. 1a). Subsequently, the PEG concentration and incubation time were optimized in banana transformation with the pUbi-GFP plasmid based on rice and wheat protocols. The highest transformation rate was observed when PEG concentration was increased to 50\% and induction time was as high as $30 \mathrm{~min}$ (Fig. 1d). The transformation efficiency was $5.6 \%$, determined by flow cytometry detection (Fig. 1e). To determine the editing efficiency of different editing methods in banana, CRIS PR/Cas9-PDS plasmids, CRISPR/Cas12a-PDS plasmids and a CRISPR/Cas9 RNP-PDS complex (RNPs) were examined using the optimized protoplast transformation protocol following the flow chart shown in Fig. 2.

\section{PEG-mediated PCR-RE assay of gene editing in banana protoplast through plasmid DNA transformation}

To explore whether our protoplast transformation system can be applied to genome editing by transferring plasmid DNAs, we designed 9 sgRNAs, of which two sgRNAs target the 2nd (target 3: OsU3p-PDSt3) and 7th exon (target 4: OsU3p-PDSt4) of the banana PDS gene, respectively. These two sgRNAs both contain recognition sites for Eco47I that can be used for PCR-RE assays (Fig. 3). Each of these 9 guided RNAs were fused with an enhanced scaffold RNA (Fig. 3a). These plasmid DNAs and the Cas9 plasmid were transformed into the banana protoplasts using the abovementioned method. DNA isolated from resulting transformed and nontransformed (control) protoplasts were used for PCR-RE assays and sequencing analysis. PCR-RE results showed that PCR product from the control sample was completely digested into two bands (t3wt-dig, t4wt-dig), but PCR products from plasmid-transformed samples were only partially digested under the same conditions (t3kodig, t4ko-dig), suggesting that the restriction enzyme site was mutated by the gene editing cassette (Fig. 4a). To further characterize the mutation type (i.e., insertion, deletion, etc.) created by Cas9, the PCR fragments undigested by Eco47I were recovered and cloned into Tblunt vectors for Sanger sequencing. Ten single clones for OsU3p- PDSt3 and three single clones for OsU3pPDSt4 were selected for Sanger sequencing. The results indicated that a 16-bp deletion was present in two cloned fragments of OsU3p- PDSt3, and all three cloned fragments of OsU3p- PDSt4 exhibited a 1-bp insertion. The insertion and the deletion started at the fourth 

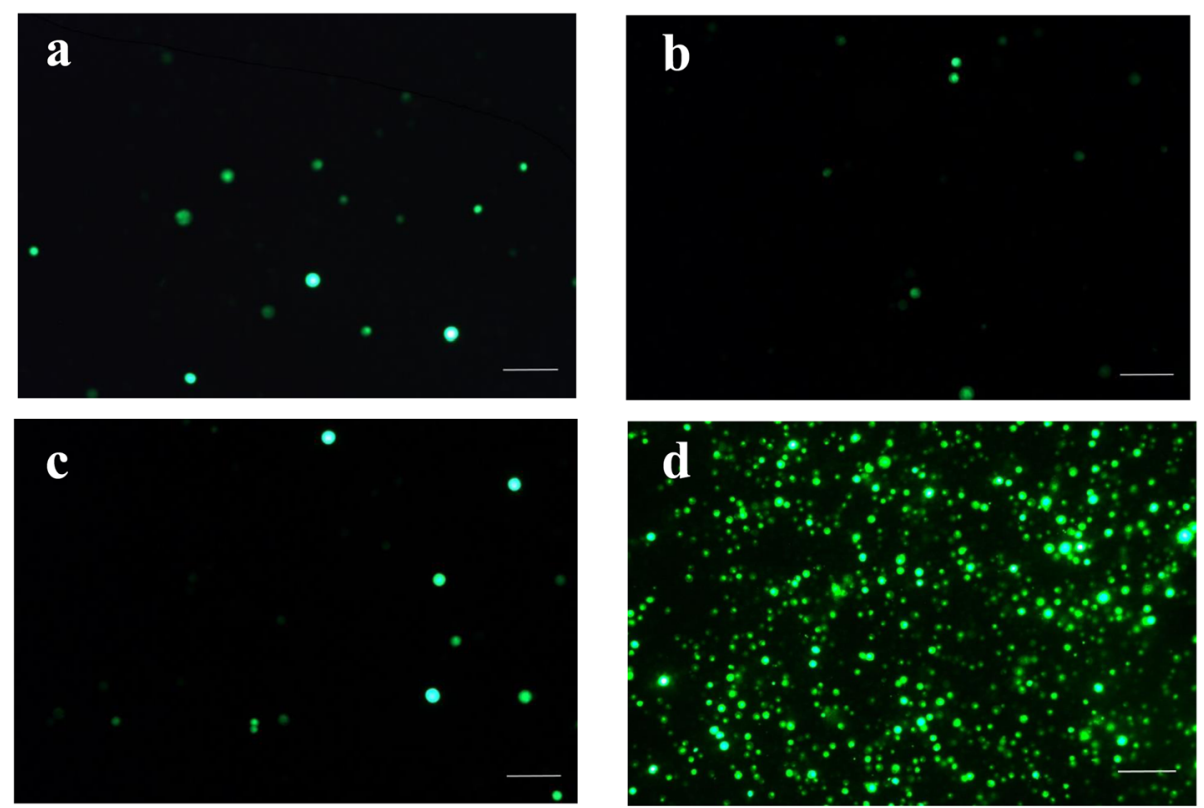

e

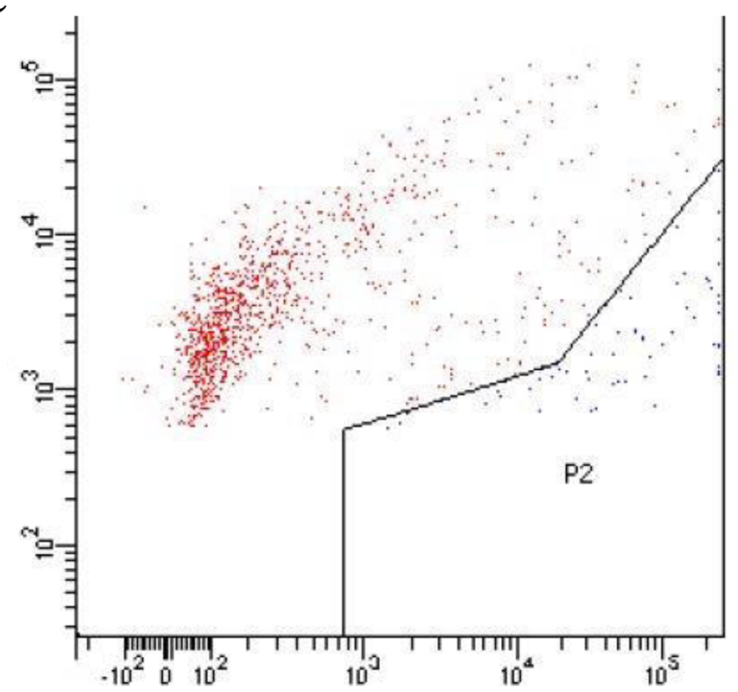

Fig. 1 Detection of GFP fluorescence in transformed protoplasts by fluorescence microscope. pUbi-GFP plasmid was used for protoplast transformation under different transformation conditions: 40\% PEG and 20 min incubation (a), 40\% PEG and 30 min incubation (b), 50\% PEG and 20 min incubation (c), 50\% PEG and 30 min incubation (d). The protoplasts from 50\% PEG with 30 min mixing were examined by flow cytometry (e). All pictures were taken after 5 days incubation in darkness. Scale bars are $75 \mu \mathrm{m}$

nucleotide from the PAM sequence (Fig. 4b). We also designed 11 sgRNAs of banana PDS gene for LbCas12a, but we did not check the results by PCR-RE assay.

\section{PCR-RE assay of gene editing in banana protoplast transformed with RNP}

To detect whether target sites mutated after RNP complexes were delivered into protoplasts, we transferred the complex of purified Cas9 proteins with targeting sgRNAs into the banana protoplasts according to the method reported by Liang $[25,26]$. We first tested whether RNPs could efficiently edit the targets in vitro prior to using them to transform protoplasts. As results show in Fig. 5a, RNPs were able to cleave the targets contained in the PCR product into two fragments. We next isolated DNA from the transformed and nontransformed protoplasts for PCR amplification. Amplified fragments containing target sequences were subjected to digestion with Eco47I. The results showed that a substantial quantity of amplified fragment could not be digested in the RNP-transformed sample, whereas amplicon from the control sample could be 


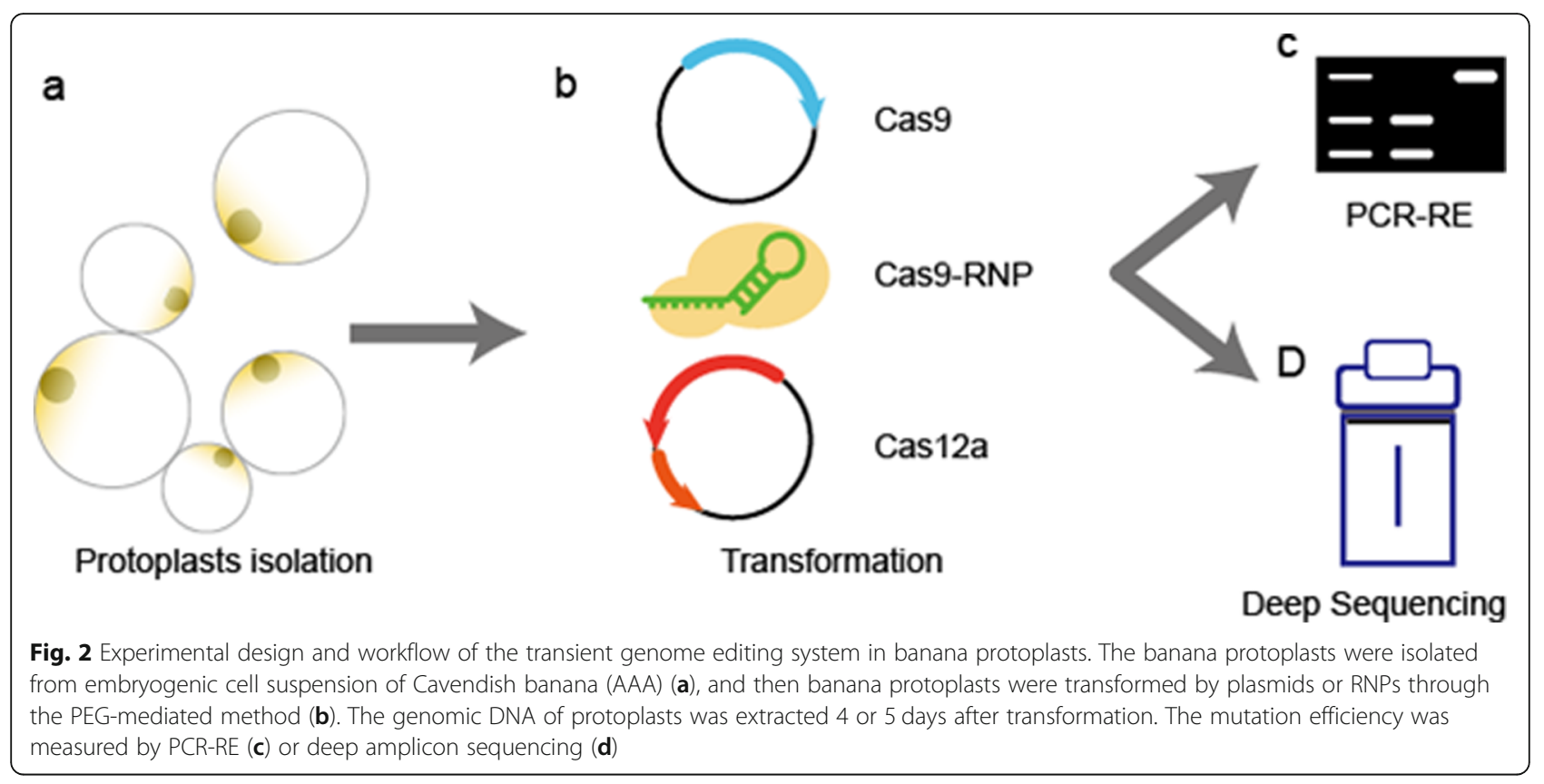

a

pOsU3-sgRNA

pUbi-LbCas12a-crRNA $\int_{\text {ubip }}^{\text {LB LbCas12a }}$

b

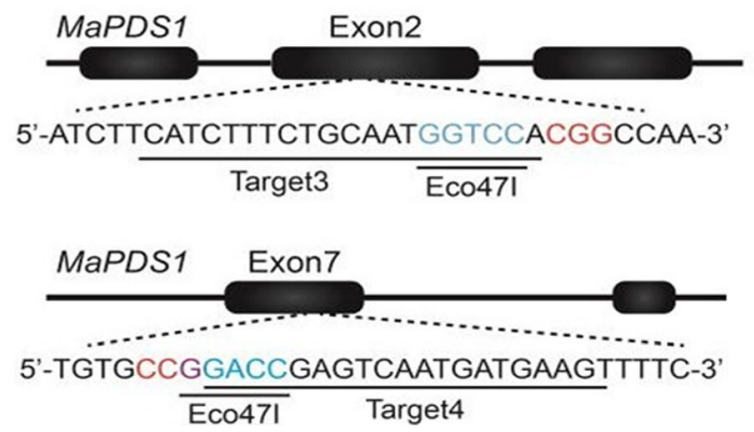

Fig. 3 Schematic representation of the vectors and targets implemented in this study. Diagrams of pUbi-Cas9, sgRNA plasmid and Cas12a vector. Ubip, ubiquitin promoter from Zea mays. Pvubip, ubiquitin promoter from Phaseolus vulgaris. CaMVter, termination sequence of cauliflower mosaic virus (a). Details of two targets on the PDS gene in banana. The positions of target 3 and target 4 on the gene and the endonuclease sites are illustrated (b). Red letters indicate PAM sequence 


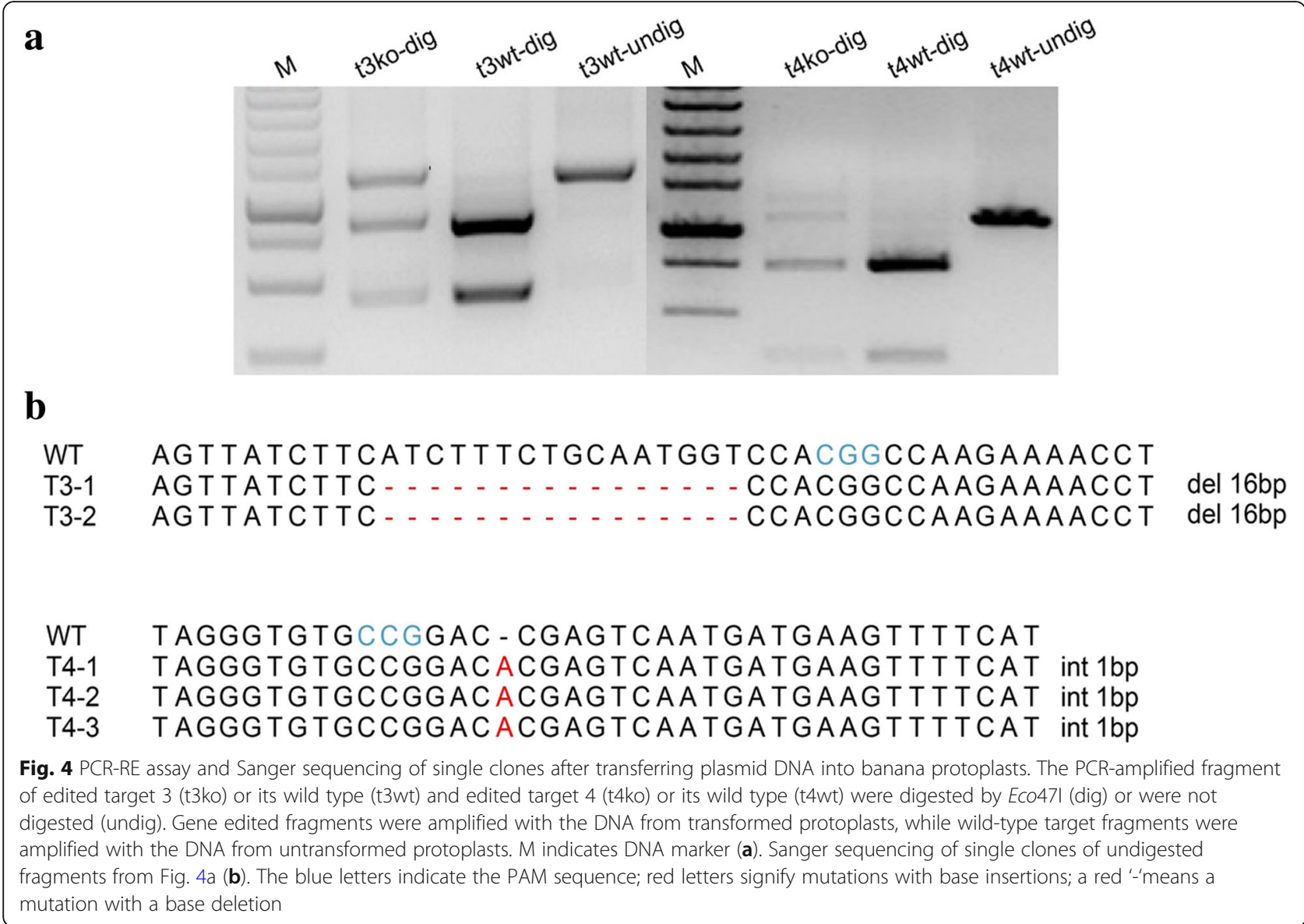

completely digested, suggesting the disruption of the Eco47 I recognition site by RNP in the RNPtransformed sample (Fig. 5 b).

\section{Deep amplicon sequencing of DNA transformation mediated by PEG in banana protoplasts}

Cavendish banana protoplasts were separately transformed with pUbi - Cas9 plasmid mixed with each of 9 sgRNA plasmids targeting PDS and each of 11 Cas12a PDS plasmids (Cas12a - PDSt1 to Cas12a - PDSt11). To examine the editing efficiency, genomic DNAs were isolated from protoplasts transformed with each sgRNA plus Cas9 or LbCas12a plasmid for deep amplicon sequencing. The sequencing results showed that 5 target sites (PDSt4, PDSt6, PDSt7, PDSt8 and PDSt9) were edited in the protoplasts transformed with sgRNAs and Cas9 plasmids. Among these edited target sites, the target site PDSt8 exhibited the highest editing efficiency of $1.04 \%$, and 320 inserts and 800 deletions out of 107,568 sequencing reads for target site PDSt8 were observed. The lowest editing efficiency of $0.18 \%$ occurred for the target site PDSt6. Mutations were observed out of 80 , 384 sequencing reads, and 147 sequencing reads contained various deletions (Additional file 1:Table S1).
For the LbCas12a system, four target sites (Cas12aPDSt1, Cas12a- PDSt7, Cas12a- PDSt9 and Cas12aPDSt10) were successfully edited. The highest editing was observed for the target site Cas12a- PDSt9 with an efficiency of $0.39 \%$, and deletion-type editing was found in 114 out of 29,008 sequencing reads for Cas12aPDSt9. In contrast, only 78 (3 insertions and 75 deletions) of 52,965 sequencing reads contained mutations created in the target site Cas12a-PDSt7, resulting in the lowest editing efficiency $(0.15 \%)$ at this target site (Additional file 2:Table S2).

\section{Deep amplicon sequencing of 9 complexes of Cas9 and sgRNAs for PEG-mediated transformation of banana protoplasts}

Cas 9 proteins combined separately with 9 sgRNAs were transformed into banana protoplasts. To examine the editing efficiency of transforming RNPs, we also performed deep amplicon sequencing of genomic DNA isolated from the protoplasts transformed with the 9 complexes of Cas 9 and sgRNAs targeting 9 different PDS sites. We have detected editing by this RNP system at five target sites, including PDS-sgRNAt2, PDS -sgRNAt4, PDS -sgRNAt6, PDS -sgRNAt7 and PDS 


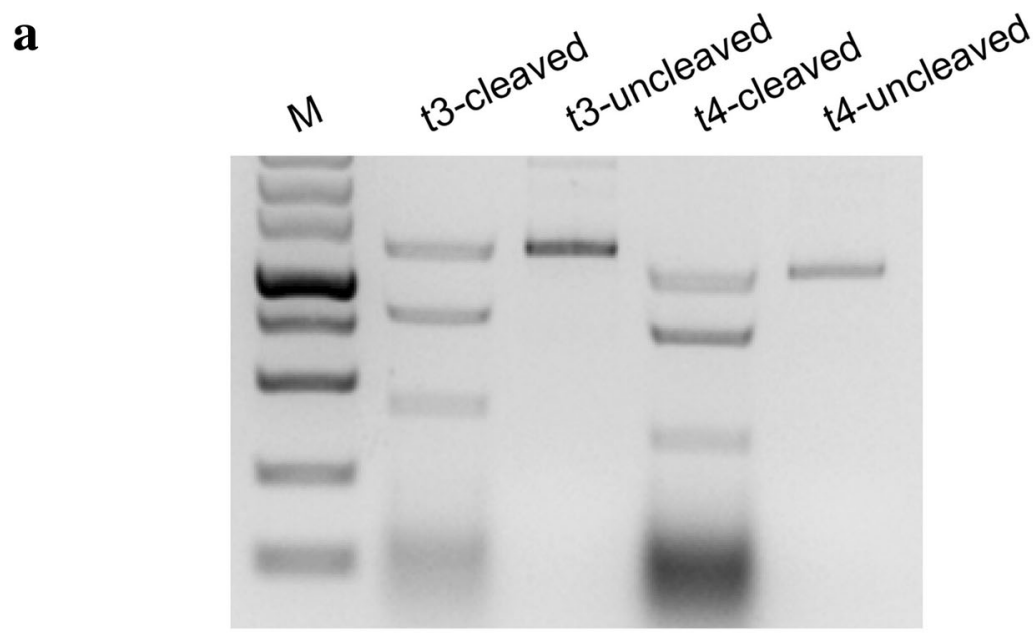

b

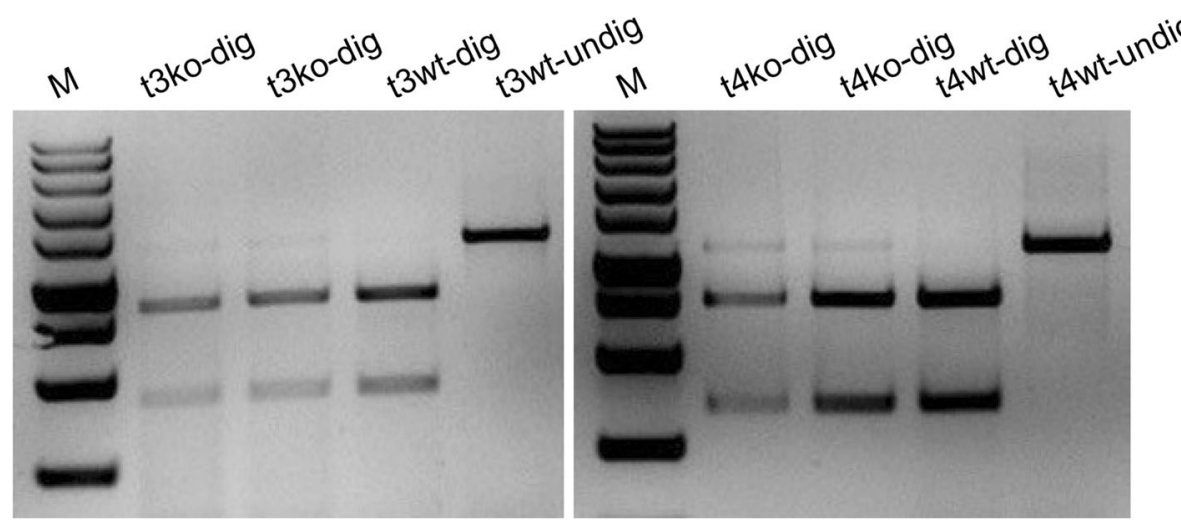

Fig. 5 RNP cleavage test in vitro and PCR-RE test after protoplast transformation. RNP cleavage test in vitro; t3-cleaved and t4-cleaved were used to cut the target 3 and target 4 PCR sequences by RNPs, while t3-uncleaved, as a control, was the sequence of target 3 PCR, and t4-uncleaved was the target 4 PCR sequence (a). ko-dig refers to Eco471 enzyme digestion of PCR amplification target sequences after RNP transformation of protoplasts; wt-dig refers to ECO471 enzyme digestion of target sequences of PCR amplification after blank transformation of protoplasts; wt-undig refers to PCR amplification after blank transformation of protoplasts, as a control. M indicates DNA marker (b)

-sgRNAt9. The target site PDS -sgRNAt9 had the lowest editing efficiency $(0.19 \%)$, including 166 insertion reads and 26 deletion reads out of 102,866 reads. The target site PDS -sgRNAt6 exhibited the highest editing efficiency $(0.92 \%)$, including 169 insertion reads and 1104 deletion reads out of 138,085 reads (Additional file 3: Table S3).

\section{Off-target detection}

To analyze the off-target effect of CRISPR/Cas9-PDS plasmids and the CRISPR/Cas9 RNP-PDS complex (RNPs) gene editing system, a potential off-target site (AGCTTCGTGTACCGCAGTAGTGG), GSMUA Achr6G21680_001, was predicted via the CRISPR-P 2.0 website. There are four base mismatches between the off-target site and the sgRNA sequence. Through deep amplicon sequencing, only one site was detected in the CRISPR/Cas9-PDS and RNPs gene editing system, and the off-target efficiency was $0.01 \%$. (Additional file 4: Table S4, Additional file 5:Table S5, Additional file 6: Table S6).

\section{Discussion}

Banana is a kind of tropical and subtropical monocotyledonous perennial herbaceous plant. Most cultivated varieties are triploid, with high fertility. As there is no seed in edible bananas, it is very difficult to achieve fine varieties with good quality and strong disease-resistance through traditional crossbreeding cultivation. However, there is a certain blindness and longer development cycle requirement with breeding new varieties by mutation breeding and mutant screening. For stable CRISPR/ Cas9 gene editing by Agrobacterium-mediated transformation in major banana cultivars, the sterility of pollen makes it difficult to remove the exogenous integrated DNA by crossing as is done with diploid plants. 
Due to the absence of exogenous DNA integrated into the genome in gene editing with CRISPR/Cas9 RNP complexes, this approach provides an effective option for banana molecular breeding by gene editing.

Woo et al. (2015) reported that CRISPR/Cas9 RNP complexes can be delivered into the protoplasts of Arabidopsis thaliana, tobacco, lettuce and rice for gene editing, and the mutation efficiency of the target in tobacco was as high as 44\% [18]. Malnoy et al.(2016) delivered RNPs into grape and apple protoplasts by PEG, and the mutation efficiency in apples reached 6.9\%, as measured by deep amplicon sequencing [19]. In 2016, Svitashev et al. directly delivered an RNP complex into immature embryos of corn by biolistic bombardment and achieved targeted gene editing with $0.69 \%$ mutation efficiency [27]. Similarly, Liang et al. (2017) developed an efficient DNA-free genome editing method in bread wheat using Cas9 RNP complexes by particle bombardment with $0.56 \%$ mutation efficiency [25]. These results indicate that the mutation efficiency of RNP complexes using PEG-mediated protoplast transformation is much higher than that using biolistic bombardment.

Since CRSPR/Cas9 gene editing technology was first reported in 2013, it has been widely used and rapidly developed. A protoplast transient transformation system can effectively detect the activity of target gene sites, providing an effective means for screening gRNA and is a very good auxiliary tool for the application of CRSPR/ Cas9 gene editing technology. In this study, banana protoplast systems were transformed with Cas9 and OsU3p- PDS, RNPs, and Cas12a-PDS. Through deep amplicon sequencing, mutation efficiency of the Cas9 system was found to be greater than that of an RNP at the same target; for example Cas9 and OsU3p- PDSt4 $(0.65 \%)>$ PDSt4-RNP $(0.27 \%)$, and Cas9 and OsU3pPDSt7 $(0.55 \%)>$ PDSt7-RNP (0.42\%), Cas9 and OsU3pPDSt9 $(0.42 \%)>$ PDSt9-RNP (0.19\%). Among 9 target sites, the highest editing efficiency of the detected Cas9 system was achieved by Cas 9 and OsU3p - PDSt8, with a mutation efficiency of 1.04\%; the highest mutation efficiency of a detected RNP system was for PDSt6-RNP, with a mutation efficiency of $0.92 \%$; the highest editing efficiency of a detected Cas12a system was for Cas12aPDSt9, with a mutation efficiency of $0.39 \%$, mostly consistent with the predicted results (Fig. 6).

Recently, CRISPR/Cas9 technology has been applied to knock out the PDS gene in banana to achieve an albino mutant [20-22] and to disrupt the MaGA20ox2 gene to obtain semidwarf banana material [23]. However, there have been no reports about transformation of banana protoplasts with RNPs or the delivery of RNPs into embryogenic cell suspension (ECS) of banana. Although it has been reported that regenerated plants can be obtained by nursing culture of banana protoplasm [28, 29], this method displays low regeneration rate, poor reproducibility, and difficult regeneration. Therefore, further research on the improvement of plant regeneration efficiency from Cas9 RNP complex transformed protoplasts is very important for the application of this technology in banana breeding. The mutation efficiency from transformation of protoplasts with RNPs is much higher than that from delivery of RNP into ECS by biolistic bombardment. There is a high potential that regeneration plants free of exogenous DNA can be achieved from banana protoplasts transformed with RNP by the PEGmediated method, as long as an increase in banana protoplast regeneration efficiency can be accomplished. This proposal has vital significance in genetic improvement of banana and generation of new non-transgenic germplasms.

\section{Conclusion}

In this study, we optimized and established a banana protoplast transformation method base on protocols for rice and wheat, which is useful for gRNA activity validation. In addition, CRISPR/Cas9, CRISPR/Cas12a and a

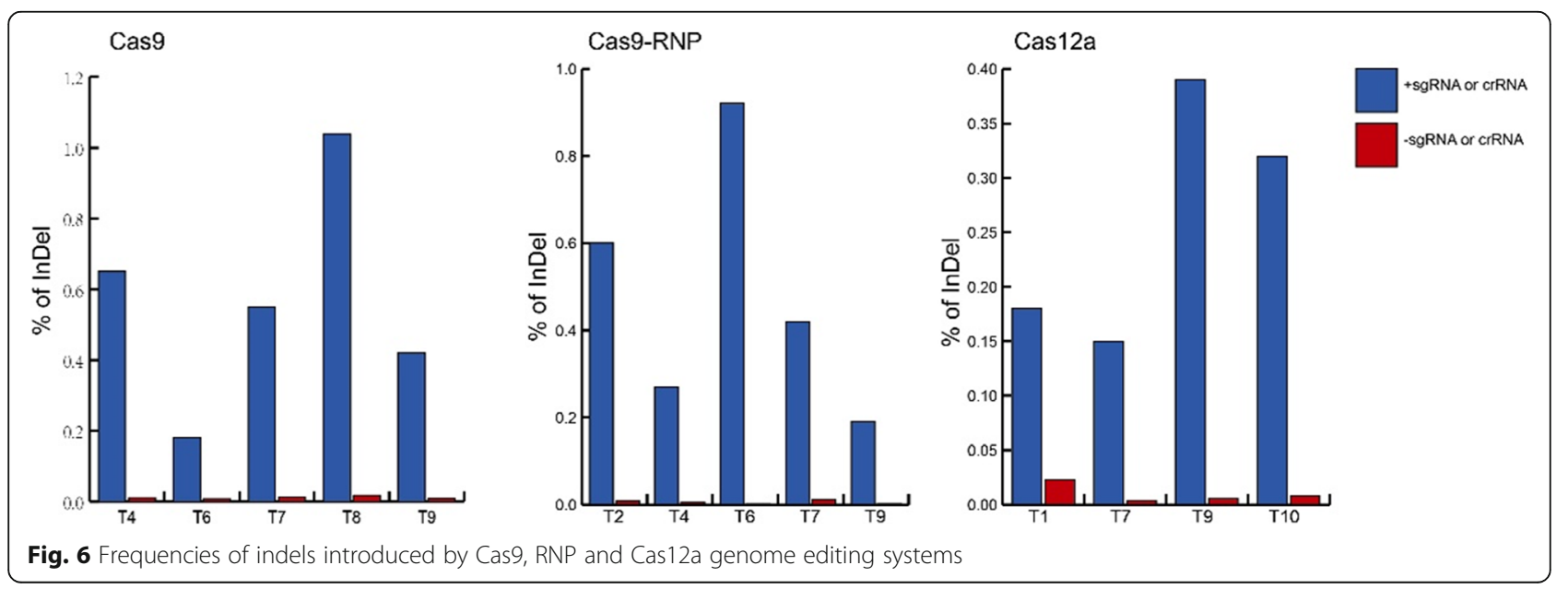


Cas9 RNP complex can successfully edit endogenous genes via an optimized protocol. The efficiency of the CRISPR/Cas9 system was greater than for the other two systems. In addition, delivering the Cas9-RNP complex into protoplasts using the PEG-mediated method shows apparent advantages compared to biolistic bombardment of ECS, and further research on plant regeneration from protoplasts is critical for successfully establishing a DNA-free gene editing system in banana.

\section{Methods}

\section{Plant material}

Plant material used in this study was Cavendish Banana (Musa spp. Cavendish; AAA Group cv. 'Baxi'); the 'Baxi' banana is one banana cultivar that has been grown in China for many years and is one of the main banana cultivars in China. We obtained male flower buds of 'Baxi' banana from the Institute of Fruit Tree Research, Guangdong Academy of Agricultural Sciences, Guangzhou, P. R. of China. ECS was induced by our laboratory in the Institute of Fruit Tree Research, Guangdong Academy of Agricultural Sciences.

\section{Banana protoplast preparation}

Small and evenly distributed subculture of ECS, cultured for approximately 10 days, was selected; the M2 medium was removed, $10 \mathrm{ml}$ of enzymatic hydrolysate $(3.0 \%$ cellulose $\mathrm{R}^{-10}, 1 \%$ segregation enzyme $\mathrm{R}-10,0.2 \%$ pectinase Y-23, $15.2 \mathrm{~g} / \mathrm{L} \mathrm{KCl}, 7.8 \mathrm{~g} / \mathrm{L} \mathrm{CaCl}, 100 \mathrm{mg} / \mathrm{L}$ MES, $10 \%$ mannitol, pH 5.7) was added; and the cells were incubated in a shaking table at $50 \mathrm{rpm} / \mathrm{min}$ for $6-8 \mathrm{~h}$. The yield of protoplasts was observed by microscope. If the enzymatic hydrolysis was sufficient, the hydrolysate was diluted with $10 \mathrm{ml}$ W5 solution and shaken for $10 \mathrm{~s}$ to separate the protoplasts. A $75-\mu \mathrm{m}$ membrane was used to filter the enzymatic solution into a round-bottom centrifugal tube. Centrifugation was performed at $100 \mathrm{~g}$ for $3 \mathrm{~min}$, and the supernatant was removed by pipette. Protoplasts were suspended in $15 \mathrm{ml} \mathrm{W5}$ solution and incubated on ice for $30 \mathrm{~min}$, and the supernatant was discarded.

\section{gRNA design and vector construction}

A total of 9 vectors targeting the PDS gene in banana were designed by SnapGene software. The OsU3p vector was digested by Bsal, and the fragments were recovered from agarose gel. The serial joint primers (Additional file 7:Table S7) were linked to the recycled OsU3p vector by T4 ligase to generate OsU3p- PDSt1 to OsU3p- PDSt9 and then used to transform E. coli DH5 $\alpha$ competent cells. After overnight culture at $37^{\circ} \mathrm{C}$, single colonies verified by sequencing were inoculated in LB liquid medium with ampicillin. After overnight culture at $37^{\circ} \mathrm{C} 220 \mathrm{rpm}$, plasmids were extracted.

\section{CRISPR/Cas12a vector construction}

A total of 11 vectors targeting the PDS gene in banana were designed by SnapGene software. The Cas12a vector was digested by Bsa1, and the fragments were recovered from agarose gel. The serial joint primers (Additional file 8:Table S8) were linked to the recycled Cas12a vector by T4 ligase to generate Cas12a- PDSt1 to Cas12a- PDSt11 and then used to transform E. coli DH5 $\alpha$ competent cells. After overnight culture at $37^{\circ} \mathrm{C}$, single colonies verified by sequencing were inoculated in LB liquid medium with kanamycin. After overnight culture at $37^{\circ} \mathrm{C}$ and $220 \mathrm{RPM}$, plasmids were extracted.

\section{Transcription of sgRNAs in vitro}

Specific primers of target sites (Additional file 9:Table S9) were designed, using OsU3p- PDSt1 to OsU3pPDSt9 plasmids as templates, amplified by the highfidelity enzyme FastPfu and purified using the EasyPure PCR Kit. Transcription of purified PCR products in vitro was performed by the NEB HiScribe ${ }^{\mathrm{m}} \mathrm{T}$ T in vitro Transcription Kit. In vitro transcription products were purified by the TIANGEN RNA Purification Kit.

\section{Protoplast transformation with plasmid or Cas9 RNP complex}

The protoplast concentration was adjusted to $2 \times 10^{6}$ $2 \times 10^{7}$ with MMG, and protoplasts were incubated on ice. $20 \mu \mathrm{g}$ plasmids were added to a 2 -ml centrifuge tube and precipitated to the bottom of the tube by centrifugation. $200 \mu \mathrm{l}$ protoplasts were added into the tube, and the contents were lightly mixed. $250 \mu \mathrm{l} 50 \%$ PEG 4000 was added and induced transformation for $30 \mathrm{~min}$ in darkness. Addition of $900 \mu \mathrm{l} \mathrm{W5}$ solution stopped transfection. The sample was centrifuged at $100 \mathrm{~g}$ for $3 \mathrm{~min}$, and supernatant was discarded. The protoplasts were resuspended with $1 \mathrm{ml} \mathrm{W5}$ and cultured in darkness at $26-28^{\circ} \mathrm{C}$.

According to the method described above, $10 \mu \mathrm{g}$ pUbiCas9 plasmid was mixed separately with $10 \mu \mathrm{g}$ of plasmids OsU3p- PDSt1 to OsU3p- PDSt9, $20 \mu \mathrm{g}$ of plasmids Cas12a- PDSt1 to Cas12a- PDSt11, Cas9 protein $(20 \mu \mathrm{g})$ and sgRNA $(20 \mu \mathrm{g})$. Banana protoplasts were separately transformed by each of the prepared samples using the PEG method and then dark-cultured. The pUbi-GFP plasmid was used for transformation as a control.

\section{PCR-RE test and sanger sequencing of single clones}

After DNA and RNP transformation, the target sites with specific endonuclease sites were selected for PCR RE tests. Genomic DNA of protoplasts was extracted. Sequences with lengths of approximately $1000 \mathrm{bp}$ containing target sites were amplified by PFU enzyme. The specific PCR products were digested by Eco 471 at $37^{\circ} \mathrm{C}$ 
for $2 \mathrm{~h}$ and analyzed on 2\% agarose gel by electrophoresis. To determine whether there were specific fragments with base mutations, the specific fragments were recovered after electrophoresis, connected to the T-blunt vector, used to transform $E$. coli DH5 $\alpha$ competent cells and then selected for Sanger sequencing of single clones.

\section{RNP cleavage in vitro}

DNA fragments containing target 3 and target 4 were amplified by PCR, purified using the EasyPure PCR Purification Kit, and eluted by RNase-free water. The cleavage reaction system in vitro was as follows: Cas9 protein $(1 \mu \mathrm{g})$, sgRNA $(1 \mu \mathrm{g})$, target fragment $(100 \mathrm{ng}), 10 \times$ Cas 9 reaction buffer (20 mM HEPES, pH 7.5, $150 \mathrm{mM} \mathrm{KCl,}$ $10 \mathrm{mM} \mathrm{MgCl} 2,0.5 \mathrm{mM}$ DTT) $2 \mu \mathrm{l}$, RNase-free water up to total volume of $20 \mu \mathrm{l}$. Samples were incubated at $37^{\circ} \mathrm{C}$ for $1 \mathrm{~h}$ and then at $65^{\circ} \mathrm{C}$ for $10 \mathrm{~min}$. Finally, samples were tested by electrophoresis on $2 \%$ agarose gel.

\section{Deep amplicon sequencing}

Banana protoplasts were transformed with prepared samples of DNA or RNP. After 4-5 days dark culture, the banana protoplasts were collected by centrifugation at 12000 RPM, and then genomic DNA was extracted with the TIANGEN DNA Extraction Kit. Deep amplicon sequencing primers (Additional file 10:Table S10, Additional file 11:Table S11) were designed, and nested PCR was performed to amplify fragments with approximate lengths of $200 \mathrm{bp}$. After gel purification, samples were sent for deep amplicon sequencing by Shanghai Shenggong Biology Co., Ltd., to determine whether there were base mutations in the target sequences and the types of mutations.

\section{Supplementary information}

Supplementary information accompanies this paper at https://doi.org/10. 1186/s12870-020-02609-8.

\footnotetext{
Additional file 1: Table S1. The results of deep amplicon sequencing of Cas9 system.

Additional file 2: Table S2. The results of deep amplicon sequencing of Cas12a system.

Additional file 3: Table S3. The results of deep amplicon sequencing of RNP system.

Additional file 4: Table S4. Off-target effects of Cas9 system.

Additional file 5: Table S5. Off-target effects of RNP system.

Additional file 6: Table S6. Primer pairs for off-target detection.

Additional file 7: Table S7. Primer pairs used to construct OsU3p-PDS.

Additional file 8: Table S8. Primer pairs used to construct Cas12a-PDS.

Additional file 9: Table S9. Primer pairs used for in vitro transcription of sgRNA.

Additional file 10: Table S10. Primer pairs used for capturesequencing of Cas9 and RNP system.
}

Additional file 11: Table S11. Primer pairs used for capturesequencing of Cas12a system.

\section{Abbreviations}

PEG: Polyethylene glycol; CRISPR: Clustered regularly interspaced short palindromic repeats; RNP: Ribonucleoprotein; PCR-RE: PCR-Restriction Enzyme; PDS: Phytoene dehydrogenase; GFP: Green fluorescent protein; ECS: Embryogenic cell suspension; sgRNA: Single-guide RNA

\section{Acknowledgements}

The authors would like to thank Caixia Gao for technical support and kindly providing the Cas9, gRNA and Cas12a vectors.

\section{Authors' contributions}

G.Y., K.C. designed research; S.W., H.Z., J.L., Q.Y., H.S., F. B., C.H., performed research; S.W., H. Z. analyzed data; S.W., H.Z.,H.H., Q.Y. wrote the paper. The authors have read and approved the manuscript.

\section{Funding}

This work was supported by the National Key R\&D Program of China (2018YFD1000302 and 2019YFD1000901), National Natural Science Foundation of China (31872939), Modern Agricultural Innovation Team Project of Guangdong Province (2018LM2150 and 2019KJ106), R\&D Plan of Guangdong Province Key Fields (2018B020202005), Science and Technology Plan Project of Guangdong Province (2015B070701011), and Guangzhou Scientific Research Plan (201904020033), Special fund for scientific innovation strategy-construction of high level Academy of Agriculture Science (R2017PY-JX001). The design of the study and collection, analysis, and interpretation of data and writing the manuscript were performed by all the authors. The funding bodies just provide the financial support.

\section{Availability of data and materials}

Deep amplicon sequencing data are available under BioProject IDs PRJNA637446 (https://www.ncbi.nlm.nih.gov/sra/PRJNA637446),

PRJNA637703 (https://www.ncbi.nlm.nih.gov/sra/PRJNA637703) and PRJNA63 7699 (https://www.ncbi.nlm.nih.gov/sra/PRJNA637699).

Ethics approval and consent to participate

Not applicable.

\section{Consent for publication}

All listed coauthors have contributed to the manuscript, reviewed the manuscript and agreed with its publication in BMC Plant Biology.

\section{Competing interests}

The authors declare that they have no competing interests.

\section{Author details}

${ }^{1}$ College of Horticulture, Hunan Agricultural University, Changsha, China. ${ }^{2}$ Key Laboratory of South Subtropical Fruit Biology and Genetic Resource Utilization (Ministry of Agriculture and Rural Affairs), Guangdong Key Laboratory of Tropical and Subtropical Fruit Tree Research, Institute of Fruit Tree Research, Guangdong Academy of Agricultural Sciences, Guangzhou, China. ${ }^{3}$ State Key Laboratory of Plant Cell and Chromosome Engineering, Center for Genome Editing, Institute of Genetics and Developmental Biology, Chinese Academy of Sciences, Beijing, China. ${ }^{4}$ University of Chinese Academy of Sciences, Beijing, China. ${ }^{5}$ Mid-Florida Research and Education Center, University of Florida, Apopka, FL, USA.

Received: 6 June 2020 Accepted: 17 August 2020 Published online: 15 September 2020

\section{References}

1. Klercker JAF. Eine method zur isolierung lebender protoplasten [J]. Ofveers vetensk Akad Forh Stock. 1892;49:463-75.

2. Cocking EC. A method for the isolation of plant protoplasts and vacuoles [J]. Nature. 1960;187:962-3.

3. Potrykus I, Shillito RD, Saul MW, Paszkowski J. Direct gene transfer state of the art and future potential [J]. Plant Mol Biol Report. 1985;3:117-28. 
4. Krens FA, Molendijk L, Wullems GJ, Schilperoot RA. In vitro transformation of plant protoplasts with Ti-plasmid DNA [J]. Nature. 1982;296:72-4.

5. Fromm M, TaylorL P, Walbot V. Expression of genes transferred into monocot and dicot plant cells by electroporation [J]. Proc Natl Acad Sci. 1985;82(17):5824-8.

6. Nishiguchi M, Langridge WHR, Szalay AA, Zaitlin M. Electroporationmediated infection of tobacco leaf protoplasts with tobacco mosaic virus RNA and cucumber mosaicvirus RNA [J]. Plant Cell Rep. 1986:5:57-60

7. Ou-Lee TM, Turgeon R, Wu R. Expression of a foreign gene linked to either a plant-virus or a drosophila promoter, after electroporation of protoplasts of rice, wheat, and sorghum [J]. Proc Natl Acad Sci. 1986; 83(18):6815-9.

8. Hauptmann RM, Ozisa-Akins P, Vasil V, Tabaeizadeh Z, Rogers SG, Horsch B, et al. Transient expression of electroporated DNA in monocotyledonous and dicotyledonous species [J]. Plant Cell Rep. 1987; 6:265-70.

9. Jones H, Ooms G, Jones MGK. Transient gene expression in electroporated solanum protoplasts [J]. Plant Mol Biol. 1989;13:503-11.

10. Hillmer S, Gilroy S, Jones RL. Visualizing enzyme secretion from individual barley (Hordeum vulgare) Aleurone protoplasts [J]. Plant Physiol. 1992;102: 279-86.

11. Zou JJ, Wei FJ, Wang C, Wu JJ, Disna R, Liu WX, et al. Arabidopsis calciumdependent protein kinase CPK10 functions in abscisic acid- and Ca2+ -mediated stomatal regulation in response to drought stress [J]. Plant Physiol. 2010;154(3):1232-43.

12. Sheen J. Signal transduction in maize and Arabidopsis mesophyll protoplasts [J]. Plant Physiol. 2001;127(4):1466-75.

13. Yoo SD, Cho YH, Sheen J. Arabidopsis mesophyll protoplasts: a versatile cell system for transient gene expression analysis [J]. Nat Protoc. 2007; 2(7):1565-72.

14. Martinho C, Confraria A, Elias CA, Crozet P, Rubio-Somoza I, Weiqel D, et al. Dissection of miRNA pathways using Arabidopsis mesophyll protoplasts [J]. Mol Plant. 2015;8(2):261-75.

15. Lee $B$, Murdoch $K$, Topping J, et al. Transient gene expression in aleurone protoplasts isolated from developing caryopses of barley and wheat [J]. Plant Mol Biol. 1989;13(1):21-9.

16. Yang JW, Fu JX, Li J, Cheng L, Li F, Dong JF, et al. A novel coimmunoprecipitation protocol based on protoplast transient gene expression for studying protein-protein interactions in Rice [J]. Plant Mol Biol Report. 2014;32(1):153-61.

17. Cao JM, Yao DM, Lin F, Jiang MYSS. PEG-mediated transient gene expression and silencing system in maize mesophyll protoplasts: a valuable tool for signal transduction study in maize [J]. Acta Physiol Plant. 2014;36(5):1271-81.

18. Woo JW, Kim J, Kwon SI, Corvalan C, Cho SW, Kim H, et al. DNA-free genome editing in plants with preassembled CRISPR-Cas9 ribonucleoproteins [J]. Nat Biotechnol. 2015;33:1162-4. https://doi.org/10. 1038/nbt.3389.

19. Mickael M, Roberto V, Min-Hee J, OK-Jae K, Seokjoong K, Jin-Soo K, et al. DNA-free genetically edited grapevine and apple protoplast using CRISPR/ Cas9 ribonucleoproteins [J]. Front Plant Sci. 2016;7:1904.

20. Hu CH, Deng GM, Sun XX, Zuo CW, Li CY, Kuang RB, et al. Establishment of an efficient CRISPR/Cas9-mediated gene editing system in Banana [J]. Sci Agric Sin. 2017;50(7):1294-301.

21. Kaur N, Alok A, Shivani KN, Pandey P, Awasthi P, et al. CRISPR/Cas9mediated efficient editing in phytoene desaturase (PDS) demonstrates precise manipulation in banana cV. Rasthali genome. Funct Integr Genomics. 2018;18(1):89-99.

22. Fatima N, Benjamin D, Jennifer K, Anthony B, Kylie S, Peter W, et al. Gene editing the phytoene desaturase alleles of Cavendish banana using CRISPR/ Cas9[J]. Transgenic Res. 2018;27:451-60.

23. Shao XH, Wu SP, Dou TX, Zhu HC, Hu CH, Huo HQ, et al. Using CRISPR/Cas9 genome editing system to create MaGA200x2 gene modified semi-dwarf banana [J]. Plant Biotechnol J. 2019;18(1):17-9.

24. Zong Y, Wang YP, Li C, Zhang R, Chen KL, Ran YD, et al. Precise base editing in rice, wheat and maize with a Cas9- cytidine deaminase fusion [J]. Nat Biotechnol. 2017:35(5):438-41.

25. Liang Z, Chen KL, Li TD, Wang YP, Zhao Q, Liu JX, et al. Efficient DNA-free genome editing of bread wheat using CRISPR/Cas9 ribonucleoprotein complexes [J]. Nat Commun. 2017;8:14261.
26. Liang Z, Chen KL, Zhang Y, Liu JX, Yin KQ, Qiu JL, et al. Genome editing of bread wheat using biolistic delivery of CRISPR/Cas9 in vitro transcripts or ribonucleoproteins [J]. Nat Protoc. 2018;13(3):413-30.

27. Svitashev S, Schwartz C, Lenderts B, Yong JK, Cigan AM. Genome editing in maize directed by CRISPR-Cas9 ribonucleoprotein complexes [J]. Nat Commun. 2016;7:13274

28. Panis B, Wauwe AV, Swennen R. Plant regeneration through direct somatic embryogenesis from protoplasts of banana (Musaspp.)[J]. Plant Cell Rep. 1993;12:403-7.

29. Matsumoto K, Oks S. Plant regeneration from protoplasts of a Brazilian dessert banana (Musa spp. AAB group)[J]. Acta Hortic. 1998; 490:455-62.

\section{Publisher's Note}

Springer Nature remains neutral with regard to jurisdictional claims in published maps and institutional affiliations.
Ready to submit your research? Choose BMC and benefit from:

- fast, convenient online submission

- thorough peer review by experienced researchers in your field

- rapid publication on acceptance

- support for research data, including large and complex data types

- gold Open Access which fosters wider collaboration and increased citations

- maximum visibility for your research: over $100 \mathrm{M}$ website views per year

At BMC, research is always in progress.

Learn more biomedcentral.com/submissions 